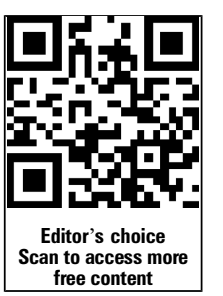
free content
fon

- Additional material is available. To view please visit the journal online (http://dx.doi. org/10.1136/emermed-201400204067).

1 Department of Medicine, Universitätsklinikum Freiburg i.Br., Freiburg i.Br., Germany 2Pharmacy Service, University Medical Center Universitätsklinikum Freiburg i.Br., Freiburg i.Br., Germany ${ }^{3}$ Universitätsklinikum Carl Gustav Carus, Dresden, Germany

${ }^{4}$ Department of Emergency Medicine, Universitätsklinikum Freiburg i.Br., Freiburg i.Br., Germany

${ }^{5}$ Institute of Medical Biometry and Informatics, Universitätsklinikum Freiburg i.Br., Freiburg i.Br., Germany

\section{Correspondence to} Dr Johannes P Borde, Abteilung Infektiologie, Universitätsklinikum Freiburg, Hugstetter Straße 55, Freiburg i.Br. D-79106, Germany; johannes.borde@web.de

Received 4 June 2014 Revised 18 August 2014 Accepted 29 August 2014 Published Online First 26 September 2014

\section{SLinked}

- http://dx.doi.org/10.1136/ emermed-2014-204220

- http://dx.doi.org/10.1136/ emermed-2015-204761

\section{CrossMark}

To cite: Borde JP, Kern WV, Hug $\mathrm{M}$, et al. Emerg Med J 2015:32:509-515.

\title{
Implementation of an intensified antibiotic stewardship programme targeting third-generation cephalosporin and fluoroquinolone use in an emergency medicine department
}

\author{
Johannes P Borde, ${ }^{1}$ Winfried V Kern, ${ }^{1}$ Martin Hug, ${ }^{2}$ Michaela Steib-Bauert, ${ }^{1}$ \\ Katja de With, ${ }^{1,3}$ Hans-Jörg Busch, ${ }^{1,4}$ Klaus Kaier $^{5}$
}

\section{ABSTRACT}

Introduction Early initiation of antimicrobial treatment for acute infection is an important task in the emergency department (ED) with a likely impact on the hospitalwide antibiotic use pattern. We implemented an antibiotic stewardship (ABS) programme focused on nontrauma emergency patients at a large university hospital centre targeting broad-spectrum cephalosporin and fluoroquinolone use.

Methods Guidelines and focused discussion groups emphasised reduced prescription of third-generation cephalosporins and fluoroquinolones and encouraged penicillins. Antibiotic consumption expressed as monthly drug density in WHO-Anatomical Therapeutic Chemical defined and locally recommended daily doses (DDD and RDD) per 100 patient days was analysed before (January 2008 to October 2011) and after starting the intervention (January 2012 to October 2013). We performed a before-and-after uncontrolled interventional study using interrupted time-series (ITS) analysis in one $E D$ to investigate $A B S$ intervention-related effects in a quasiexperimental research setting.

Results The mean monthly total antibiotic use density declined from 111 RDD (138 DDD) per 100 patient days before the intervention to 86 RDD (128 DDD) per 100 patient days after starting the intervention. Among the different antibacterial drug classes, the consumption of third-generation cephalosporins showed the largest reduction and dropped significantly by $-68 \%$ between preintervention and postintervention periods. Using the RDD dataset, ITS confirmed a highly significant postintervention change in level of third-generation cephalosporins $(-15.2,95 \% \mathrm{Cl}(-24.08$ to -6.311$))$ and a corresponding increase in the use of aminopenicillin/betalactamase inhibitor formulations $(+6.6,95 \% \mathrm{Cl}(4.169$ to 9.069$))$. The drug use densities for fluoroquinolones and for overall antibiotics declined, however, the postinterventional level changes missed statistical significance-overall $(95 \% \mathrm{Cl}(-39.99$ to 0.466$)$, fluoroquinolones $95 \% \mathrm{Cl}(-11.72$ to 4.333)).

Conclusions An intensified ABS programme using non-restrictive tools targeting third-generation cephalosporin and fluoroquinolone use in the setting of a large academic hospital emergency medicine department is feasible and effective. The intervention may serve as a model for other emergency medicine departments at hospitals with a similar structure and baseline situation.

\section{Key messages}

What is already known on this subject Earlier studies have demonstrated that restricting use of third-generation cephalosporins and fluoroquinolones is linked to a decreasing incidence of gram-negative resistant bacteria producing extended betalactamases (ESBL), infections due to C.difficile and methicillin-resistant Staphylococcus aureus (MRSA) infections. The feasibility and sustainability of an antibiotic stewardship programme targeting third-generation cephalosporin and fluoroquinolone consumption, using persuasive tools rather than restrictive tools, has not been evaluated before in the setting of large emergency medicine department.

\section{What this study adds}

An intensive antibiotic stewardship programme targeting third-generation cephalosporin and fluoroquinolone prescription, using persuasive rather than restrictive tools, was implemented in a large academic German emergency department. Interrupted time series (TS) analysis in this quasiexperimental research setting demonstrated reduced cephalosporin and fluoroquinolone drug use densities by $>20 \%$ with sustained performance. Interrupted Time $\mathrm{S}$ analysis is the statistic tool of choice to investigate interventionrelated effects of $A B S$ programmes in a quasiexperimental research-setting.

\section{INTRODUCTION}

Increasing bacterial resistance is a major threat to patients and healthcare systems worldwide. The causes of antibiotic resistance are complex, but include heavy use of antibiotics, both in humans and animals. ${ }^{1}$ An essential component in the strategies to reduce antimicrobial resistance is to limit overaggressive use of antibiotics in the hospital and primary care setting. ${ }^{23}$ Earlier studies have demonstrated that a reduction in aminoglycoside prescription is linked to a decreasing incidence of Gram-negative resistant bacteria. ${ }^{4}$ Similar findings are reported for the restriction of third-generation cephalosporins $^{6}{ }^{7}$ and fluoroquinolones. ${ }^{7} 8$ A reduction of infections due to Clostridium 
difficile, ${ }^{9-11}$ methicillin-resistant Staphylococcus aureus ${ }^{12-14}$ infections and Gram-negative bacteria producing extendedspectrum betalactamases $(\mathrm{ESBL})^{15-17}$ could be achieved by effective antibiotic stewardship (ABS) interventions. Reducing the antibacterial drug use density for the inpatient service is challenging, but has been successfully demonstrated in several interventional studies over the last 20 years. ${ }^{18} 19$ The long-established Anglosaxon model of large interdisciplinary emergency medicine departments is increasingly being set up in Germany. Staffing, the introduction of a defined curriculum for board-reviewed emergency medicine physicians and identifying specific quality-of-care standards are subject to the current vital discussion. The emergency medicine department plays an essential role in patient triage and initiating early effective therapies for admitted patients with acute and serious conditions. As in trauma care, tools have been developed for the triage of medical patients who might be in need of specific therapy, for example, anti-infective therapy, but there is only little known about the feasibility and effectiveness of specific ABS-programmes targeting antibiotic consumption in emergency departments (ED) by using core ABS tools. However, several studies underscore the demand for such programmes by detecting a rather poor compliance to current infectious disease guidelines in such settings and indicating an overuse of antibiotic substances, notably, broad-spectrum antiinfectives. $^{20-23}$ Among the most frequently diagnosed and treated infections are community acquired pneumonia (CAP), urinary tract infections (UTI) and skin and soft tissue infections. Regarding the management of acute respiratory infections in the $\mathrm{ED}$, treatment decisions can be facilitated by procalcitoninguided algorithms. ${ }^{24-28}$ Computerised decision support systems, ${ }^{29}$ restrictive strategies aiming at a reduction in ciprofloxacin use ${ }^{23}$ and even computer kiosk-expedited management of UTIs have been evaluated with moderate effects on antibiotic prescriptions $^{30}$ in the ED. Optimal selection of the targeted antibacterial drugs needs careful review of local resistance, drug use data and strategic decisions that take individual clinical and ecological (collateral damage) aspects into account. Given the trends for more adverse ecological effects of broad-spectrum cephalosporin versus penicillin use, we planned a before-and-after uncontrolled interventional study using interrupted time-series (ITS) analysis in one ED to investigate ABS intervention-related effects in a quasiexperimental research setting aiming at a reduction of third-generation cephalosporin and fluoroquinolone use in medical emergency patients.

\section{MATERIAL AND METHODS \\ Study design}

Briefly, our study was designed as an ITS to investigate intervention-related effects of ABS programmes in a quasiexperimental research setting. There was no formal calculation on the number of patients needed to include in the study. Antibiotic use data were gathered on a monthly basis from the hospital pharmacy service representing the antibiotic consumption of this clinical department. Written prescriptions handed out for outpatient pharmacies were not included in our study. The number and length of stay of all patients seen in the ED were used to calculate normalised consumption values per 100/ patient days. The ethics committee was notified about the trial -formal approval was not required, because the project was based on epidemiological data. Research involving human subjects, human material, specific human or personalised data was not carried out.

\section{Setting}

Freiburg University Hospital is a 1600-bed academic teaching hospital and tertiary care referral centre, with all major clinical services and departments including renal, lung, heart, haematopoetic stem cell transplant and a level 1 trauma centre. The ED has a 39-bed capacity with four emergency rooms, 10 intermediate care beds equipped with advanced monitoring capabilities and 25 standard care beds. The non-trauma section of the emergency medicine department is operated by three shifts; there are 17 full physician appointments. There are eight on a permanent basis and nine on rotation. During day shifts, there are three internal medicine residents, one internal medicine attending physician and one neurology resident present. During night shifts, there are two internal medicine residents plus one attending physician in service. Each patient is evaluated within $24 \mathrm{~h}$ for being admitted or treated on an outpatient basis. In 2013, there were about 38000 patient contacts in total, $60 \%$ trauma patients and $40 \%$ medical service patients. Overall, approximately $35 \%$ of the patients were admitted for in-hospital treatment. The patients were triaged according to the emergency severity index (ESI). In 2013, 2.5\% of the patients were scored as ESI 1, 13\% were categorised as ESI 2, 37.5\% ESI 3 and $47.5 \%$ were assigned to ESI 4 and 5, that is, about $15.5 \%$ of all patients required an immediate life-saving intervention or were in a high-risk situation.

\section{Antibiotic policy and stewardship programme}

Locally consented guidelines for the most frequent indications and acute infections were first available in written formats in 2006. In the following years, the overall hospital-wide antibiotic use density remained stable. However, the use of thirdgeneration cephalosporins and fluoroquinolones increased. Ceftriaxone was one of the most frequently prescribed antibiotic drug and became the first-line drug for treating CAP. Due to increasing rates of enteric bacteria-producing ESBL and vancomycin-resistant enterococci, internal guidelines were revised in October 2011. An intensified ABS programme targeting cephalosporin and fluoroquinolone use on medical wards was initiated and included the medical section of the ED.

The programme included the following components: guideline revision, information, education, intensified infectious disease consultations and standardised treatment protocols. Briefly, we started short-team briefings led by an infectious disease specialist, emphasised the need for infectious disease consultations in the ED by dedicated infectious disease physicians providing academic detailing and feedback. Fluoroquinolone and cephalosporin use, especially the use of third-generation cephalosporins, was discouraged; the use of penicillins was encouraged. The main replacement drug for ceftriaxone was ampicillin/sulbactam. The revised internal guidelines now recommended ampicillin/sulbactam formulations as first-line antibiotics for CAP (the 'Standard Operating Procedure' CAP is available from the author on request). Additionally, the use of combination therapy, in particular, including fluoroquinolones, was discouraged. Further, the 2006 established internal infectious disease guidelines covering a broad spectrum of indications were revised and made available in written, pocket-sized formats and accessible on the hospital intranet as well. Our initial idea was to test whether a reduction by $>20 \%$ in the prescription of both cephalosporins and fluoroquinolones would be possible within 12-24 months, without increasing overall use densities. The number of formal bedside infectious disease emergency consultations remained constant over time, but the number of kerbside and telephone infectious disease consultations increased by $10 \%$ 
Table 1 Comparison of mean monthly drug use density values expressed as DDD or RDD per 100 patient days in the medical emergency department preintervention and postintervention (before/after analysis using $t$ test)

\begin{tabular}{|c|c|c|c|c|c|c|c|c|}
\hline \multirow[b]{2}{*}{ Drug class } & \multicolumn{4}{|c|}{ DDD/100 } & \multicolumn{4}{|c|}{ RDD/100 } \\
\hline & Pre- & Post- & Per cent change & $\mathrm{p}$ Value & Pre- & Post- & Per cent change & $\mathrm{p}$ Value \\
\hline Cephalosporins & 41 & 31 & -24 & $<0.01$ & 39 & 22 & -42 & $<0.001$ \\
\hline $3^{\circ}$ Cephalosporins & 34 & 11 & -68 & $<0.001$ & 34 & 11 & -68 & $<0.001$ \\
\hline $1^{\circ} / 2^{\circ}$ Cephalosporins & 7 & 20 & 214 & $<0.001$ & 4 & 11 & 202 & $<0.001$ \\
\hline Penicillins & 19 & 39 & 99 & $<0.001$ & 18 & 22 & 22 & $<0.01$ \\
\hline Piperacillin \pm BLI & 14 & 11 & -22 & $<0.001$ & 16 & 12 & -22 & $<0.001$ \\
\hline Aminopenicillins+BLI & 2 & 22 & 835 & $<0.001$ & 1 & 8 & 782 & $<0.001$ \\
\hline Narrow-spectrum penicillins & 3 & 6 & 74 & 0.11 & 1 & 2 & 63 & 0.11 \\
\hline Carbapenems & 6 & 3 & -47 & $<0.001$ & 4 & 2 & -49 & $<0.001$ \\
\hline Fluoroquinolones & 21 & 17 & -22 & $<0.05$ & 19 & 14 & -26 & $<0.05$ \\
\hline Aminoglycosides & 0.5 & 0.3 & -31 & 0.34 & 0.4 & 0.2 & -31 & 0.34 \\
\hline Glycopeptides & 2 & 1 & -67 & $<0.001$ & 2 & 1 & -67 & $<0.001$ \\
\hline Macrolides/Clindamycin & 40 & 32 & -20 & $<0.05$ & 22 & 19 & -12 & 0.22 \\
\hline Total & 138 & 128 & -7 & 0.24 & 111 & 86 & -23 & $<0.001$ \\
\hline
\end{tabular}

$\mathrm{BLI}$, bealactamase inhibitor; DDD, defined daily doses; RDD, recommended daily doses.

between preintervention and postintervention, and there were many more briefings and interdisciplinary conferences including infectious disease physicians postintervention.

\section{Outcome data}

Antibiotic use data were gathered on a monthly basis from the hospital pharmacy service-before (January 2008 to October 2011) and after starting the intervention (November 2011 to October 2013). Antibiotic use density was expressed as WHO-ATC DDD, and doses adapted to local guidelines RDD, normalised per 100 patient (occupied beds) days to account for discrepancies between DDD for penicillins and commonly RDD in hospital practice. Case-mix indices, the number of patient cases and the number of admissions were obtained from the hospital administration.

\section{Statistical analysis}

The effect of the intervention on drug use density (in RDD per 100 patient days) was analysed using ITS with the period between January 2008 through October 2011 as preintervention and November 2011 through October 2013 as postintervention

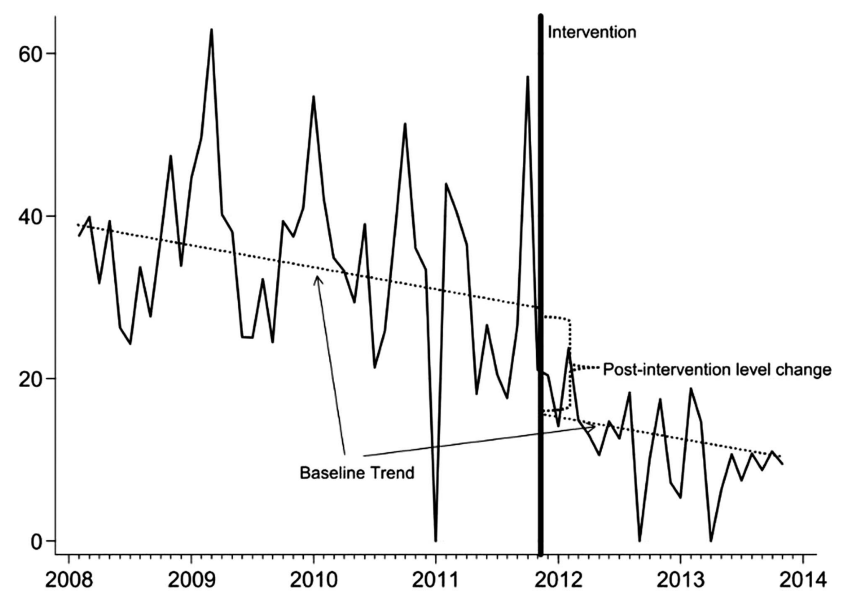

Figure 1 Drug use densities for third-generation cephalosporins (left scale recommended daily doses/100 patient days) indicating a strong postintervention level change. periods. Monthly antibiotic use data were used for the analysis. Basically, ITS allows accounting for two effects of the intervention: a level-effect or a slope-effect. As our data indicate a level change (for trend change calculations see online supplementary table S1), our regression model to analyse the use of antibiotic $\mathrm{Y}$ over time $t$ in a single setting was specified accordingly:

$$
Y_{t}=\beta_{0}+\beta_{1} \times \text { time }_{t}+\beta_{2} \times \text { intervention }_{t}+u_{t} .
$$

For introduction to the concept of ITS in the context of measuring intervention-related effects in antibiotic consumption we recommend the following references. ${ }^{31-34}$ In detail, $\beta_{0}$ determines the baseline level of the respective antibiotic use in January 2008, $\beta_{1}$ determines the baseline linear trend in the use of the respective antibiotic over the entire study period. $\beta_{2}$ determines the level-effect after the intervention. $\mathrm{u}_{\mathrm{t}}$ represents the error term. All Analyses were carried out using Stata V.12 (StataCorp, College Station, Texas, USA). We applied linear regressions with Newey-West SE with a maximum lag of 2 to be considered in the autocorrelation structure.

Descriptive statistical analysis of the data in DDD or RDD per 100 patient days was performed by using Microsoft Excel software. Heteroskedastic $t$ test was applied to compare the mean monthly drug use densities before and after the intervention.

\section{RESULTS}

The total antibiotic use density decreased in the postintervention period (for overview see figure 2 and table 1). The mean monthly drug use density values preintervention were 111 RDD (138 DDD) per 100 patient days. Following the start of the stewardship programme, these values declined by $-22 \%$, if RDD per 100 patient days was considered $(-7 \%$ for DDD/100) (table 1$)$. However, the postinterventional level changes missed statistical significance in the ITS model (95\% CI (-39.99 to 0.466)).

As intended and expected, strong effects associated with the intervention were observed for cephalosporins and penicillins. The consumption of third-generation cephalosporins declined substantially (figure 1 , table 1 ) from 34 to $11 \mathrm{RDD} / 100$ $(p<0.001)$, whereas, the use of ampicillin/sulbactam increased almost 10 -fold $(p<0.001)$. The ITS confirmed that most of the 
Original article

first/second-generation cephalosporins

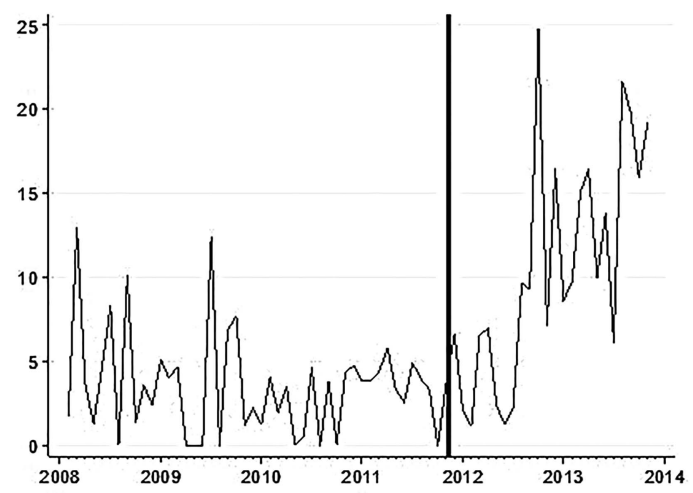

Carbapenems

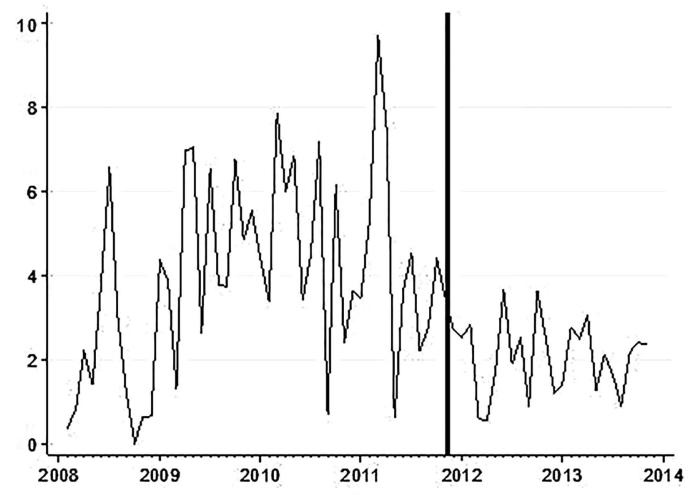

BSP (Broad Spectrum)

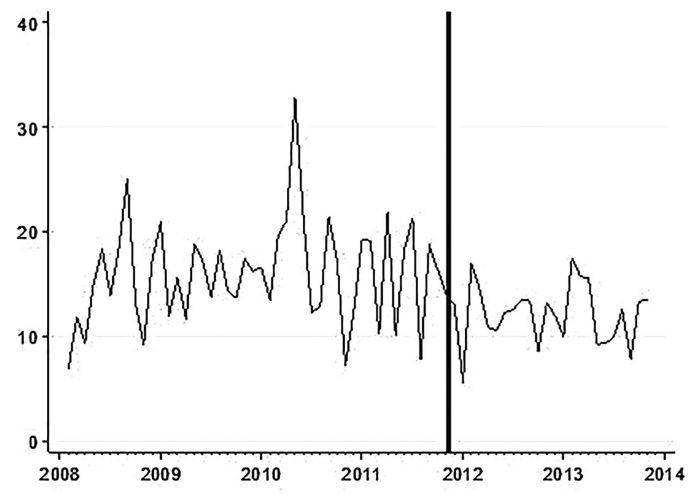

Penicillins (Narrow Spectrum)

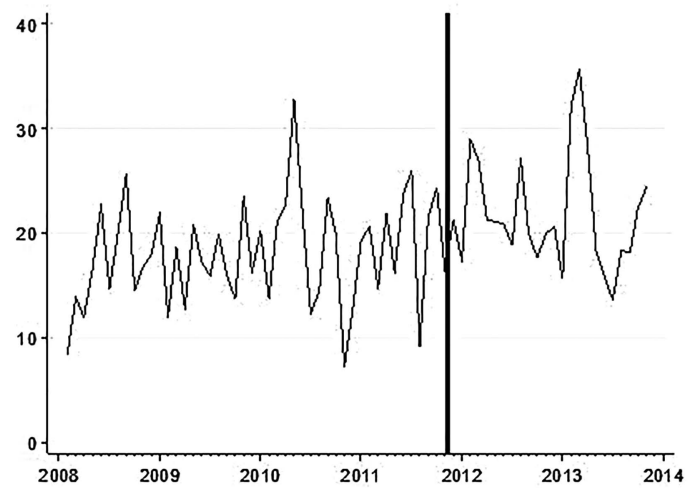

Third-generation cephalosporins

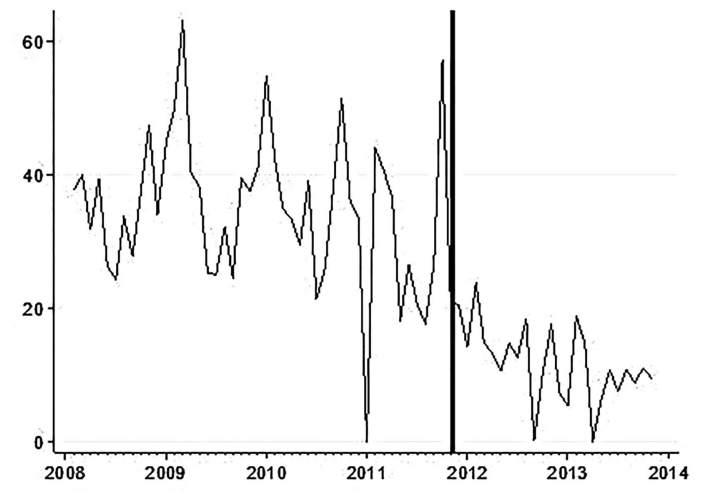

Aminopenicillins/BLI

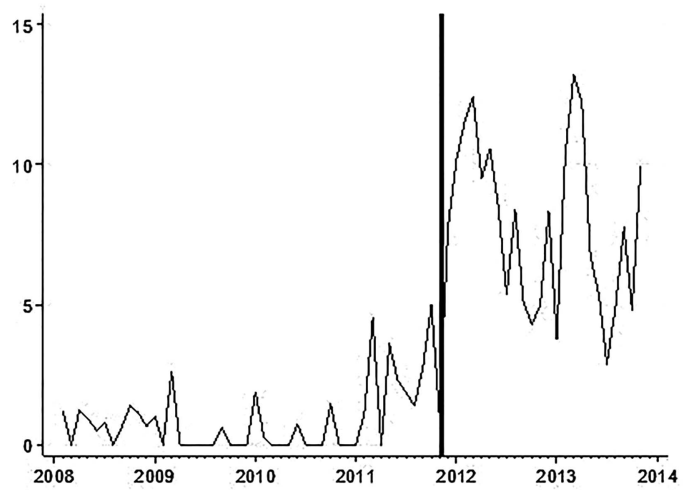

Fluorquinolones:

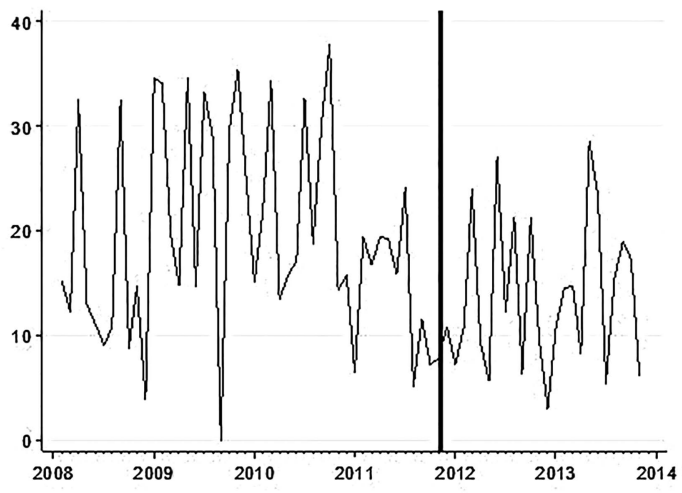

Total antibiotic use

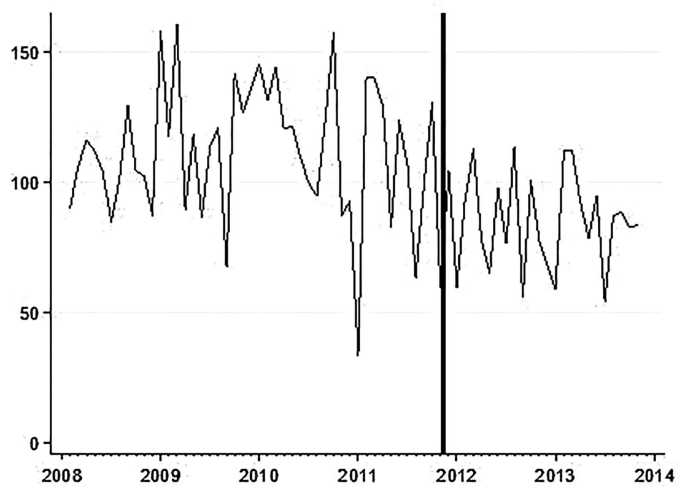

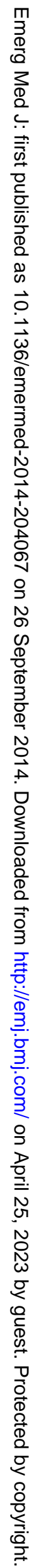

Figure 2 Run-charts for all anti-infective substances.

512

Borde JP, et al. Emerg Med J 2015;32:509-515. doi:10.1136/emermed-2014-204067 
decline of third-generation cephalosprins was, in fact, attributable to the intervention (table 2) with an intervention-related decrease of $15.2 \mathrm{RDD} / 100$ (95\% CI (-24.08 to -6.311$)$ ). By contrast with third-generation cephalosporins, other cephalosporins were increasingly prescribed following the intervention. The mean monthly use density values for first-generation and second-generation cephalosporins increased twofold, and this increase was almost entirely associated with the intervention (table 2).

There was no negative impact of the intervention in terms of increasing use of broad-spectrum betalactams (piperacillin/tazobactam and carbapenems), fluorquinolones or aminoglycosides (table 1, figure 2). The consumption of piperacillin/tazobactam and/or carbapenems showed a decreasing rather than increasing level effect postintervention. Fluoroquinolone use density declined over the observation period $(>20 \%)$, the level change between preintervention and postintervention for fluoroquinolone use did not reach statistical significance $(95 \% \mathrm{CI}(-11.72$ to 4.333$))$.

\section{DISCUSSION}

We demonstrated that an intensified ABS programme targeting third-generation cephalosporin and fluoroquinolone use in the setting of a large academic hospital ED is feasible and effective. ITS confirmed a highly significant postintervention level change of third-generation cephalosporins and a corresponding increase in the use of aminopenicillin/betalactamase inhibitor formulations. The drug use densities for fluoroquinolones and for overall antibiotics declined, however, both results missed statistical significance. ITS analysis is the statistic tool of choice to investigate intervention-related effects of ABS programmes in a quasiexperimental research setting. ${ }^{19}$

Our programme was focused on medical service patients including the ED, which is the interface between outpatient and in-hospital care and is, therefore, highly relevant for acute therapeutic decisions, including drug choices in acute infections. We were interested in demonstrating the success of our programme in the $\mathrm{ED}$, since we considered its effect to be essential for having a strong impact on the consumption in other medical service areas of this hospital. The programme itself used established components and tools of $\mathrm{ABS}$ interventions, such as revision, consensus and dissemination of local guidelines, education and feedback with discussion. Due to continuing efforts, we achieved sustainability over 2 years, however, changes in hospital-wide resistance patterns and data on possible changes in the incidence of nosocomial infections are not yet available; this is a major limitation of the study. It will be important to evaluate these outcome data 'medical service-wide', since the relatively short length of stay in the ED, the time elapsing between antibiotic therapy initiation and selection of a nosocomial superinfection with resistant micro-organisms makes it difficult to show a within-department impact on such endpoints. The present study is another example for the feasibility of ABS interventions in acute care hospitals, even in the setting of a 'high throughput' department. Many previous studies have focused on reduced cephalosporin and fluoroquinolone prescriptions and have shown variable results regarding overall consumption, effects on bacterial resistance ${ }^{14} 17$ and $C$ difficile infections. ${ }^{7} 10$ 11 It remains unknown how large, radical and how sustained the reduction of cephalosporins needs to be in order to exert durable effects on bacterial resistance and C difficile infections. Further, it is worth to speculate, which of the 'replacement' drug classes are optimal or rather negatively interfere with potential positive ecological effects in ABS programmes that successfully reduce cephalosporin prescriptions. We believe that more experience, with targeted ABS programmes

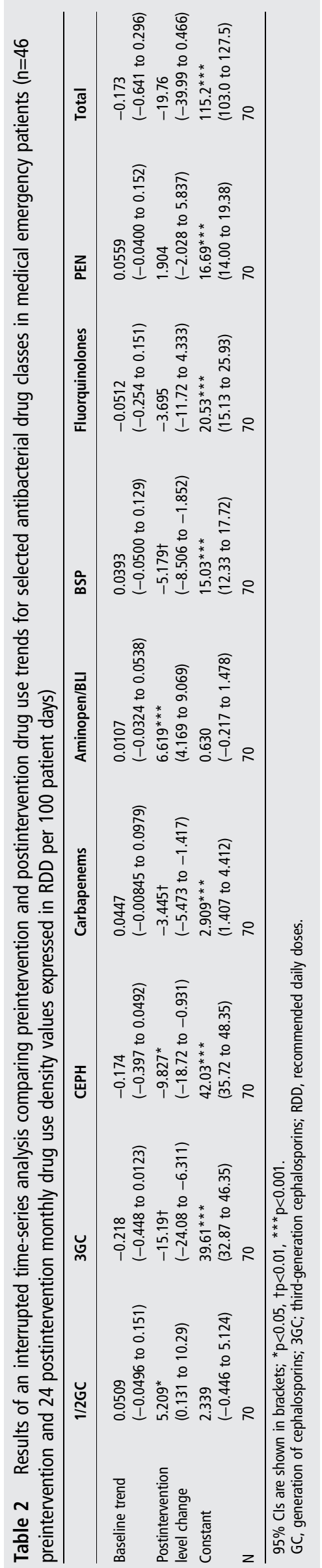


and controlled before-and-after studies is needed to address these questions properly.

Our study has several limitations. We did not use a control department against which the observed trend changes could be evaluated, and it cannot be excluded that there were some unrelated changes occurring independent from the intervention. Internal controls were unavailable, due to the unique nature of a single ED at this hospital and its influence on the prescribing on wards to which the patients were admitted. Second, although case-mix indices remained similar throughout the observation period, we cannot be sure that disease severity and the distribution of admission diagnoses remained unchanged over time. Third, we did not systematically follow-up individual patients and, therefore, cannot provide data confirming similar patient outcomes preintervention and postintervention, or data regarding intervention adherence and prescription quality. In an analysis of medical service-wide data from January 2008 to March 2013, we documented unchanged case-mix indices, discharge statistics, including in-hospital mortality and time-to-discharge, respectively, with length-of-hospital-stay. ${ }^{27}$ From March 2013 to October 2013, these crude outcome estimates remained, furthermore, roughly unchanged. We, therefore, believe that adverse outcomes of our intervention are very unlikely, although there are no specific data available on the ED level.

We were somehow surprised to see a decreasing use of carbapenems and broad spectrum pencillins (BSPs) (piperacillin/tazobactam), with significant postinterventional level changes in the ITS. These drug classes were not primary target drugs of our intervention. We hypothesise that the decreasing use density of this non-target drug classes were the results of facilitated general discussions and ward rounds with dedicated infectious disease consultants. We used persuasive rather than restrictive tools to alter the antibiotic policy in the department and were able to achieve our pursued reduction of cephalosporins and fluoroquinolones by $>20 \%$, however, the level change of fluoroquinolone prescriptions was not significant.

Of note, a small trend of fewer fluoroquinolone prescriptions was already observed prior to the programme initiation. This trend might, in part, have been related to a decision taken early in 2011 to abandon fluoroquinolone prophylaxis in haematology-oncology patients and was then intensified by reducing combination therapies including fluoroquinolones postintervention. We speculate, that fluoroquinolone consumption has reached a baseline usage in the framework of unaltered local guidelines for UTI, which can further be influenced only by specifically addressing a change in guidelines for this indication. The focus on persuasive rather than restrictive tools may be important to mention since drug restriction in the emergency or intensive care setting might have been more effective, but would not have been accepted in our hospital. Non-restrictive methods to alter antibiotic policies and to influence rates of antimicrobial resistance appear to work. However, we hypothesise that the time needed to achieve the defined goals might be longer. $^{19}$

\section{CONCLUSION}

In conclusion, an intensified ABS programme using nonrestrictive methods targeting third-generation cephalosporin and fluoroquinolone use in the setting of a large academic hospital ED is feasible and effective. This intervention may serve as a model for other EDs at hospitals with a similar structure and baseline situation.
Contributors JPB wrote the manuscript, he was involved in the ID consultant service and performed descriptive statistical analysis of the data. KK did all advanced statistical work and drafted the statistical section of the manuscript. MS-B designed and adopted the database system for ABS purposes. H-JB implemented and discussed internal guidelines for the emergency medicine department. $\mathrm{MH}$ provided prescription and consumption data from the pharmacy service. WVK and KdW were planning the project and designed the ABS interventions. WVK supervised the clinical ID consultant service.

Funding This study was supported in part by internal funds from the Department of Medicine. WVK and KdW were supported in part by a grant from the Federal Ministry of Health (BMG grant IIA5-2011-2511FSB340).

\section{Competing interests None.}

Provenance and peer review Not commissioned; externally peer reviewed.

\section{REFERENCES}

1 Laxminarayan $R$, Duse $A$, Wattal $C$, et al. Antibiotic resistance-the need for global solutions. Lancet Infect Dis 2013;13:1057-98.

2 Dellit TH, Owens RC, McGowan JE, et al. Infectious Diseases Society of America and the Society for Healthcare Epidemiology of America guidelines for developing an institutional program to enhance antimicrobial stewardship. Clin Infect Dis 2007:44:159-77.

3 Tamma PD, Cosgrove SE. Antimicrobial stewardship. Infect Dis Clin North Am 2011:25:245-60.

4 Young EJ, Sewell CM, Koza MA, et al. Antibiotic resistance patterns during aminoglycoside restriction. Am J Med Sci 1985;290:223-7.

5 Gerding DN. Antimicrobial cycling: lessons learned from the aminoglycoside experience. Infect Control Hosp Epidemiol 2000;21:S12-17.

6 Kaier K. Economic implications of the dynamic relationship between antibiotic use and hospital-acquired infections. Value Health 2012;15:87-93.

7 Dancer SJ, Kirkpatrick P, Corcoran DS, et al. Approaching zero: temporal effects of a restrictive antibiotic policy on hospital-acquired Clostridium difficile, extended-spectrum $\beta$-lactamase-producing coliforms and meticillin-resistant Staphylococcus aureus. Int J Antimicrob Agents 2013;41:137-42.

8 Livermore DM, Hope R, Reynolds R, et al. Declining cephalosporin and fluoroquinolone non-susceptibility among bloodstream Enterobacteriaceae from the UK: links to prescribing change? J Antimicrob Chemother 2013;68:2667-74.

9 Kaier K, Hagist C, Frank U, et al. Two time-series analyses of the impact of antibiotic consumption and alcohol-based hand disinfection on the incidences of nosocomial methicillin-resistant Staphylococcus aureus infection and Clostridium difficile infection. Infect Control Hosp Epidemiol 2009;30:346-53.

10 Pépin J, Saheb N, Coulombe M-A, et al. Emergence of fluoroquinolones as the predominant risk factor for Clostridium difficile-associated diarrhea: a cohort study during an epidemic in Quebec. Clin Infect Dis 2005;41:1254-60.

11 Talpaert MJ, Gopal Rao G, Cooper BS, et al. Impact of guidelines and enhanced antibiotic stewardship on reducing broad-spectrum antibiotic usage and its effect on incidence of Clostridium difficile infection. J Antimicrob Chemother 2011;66:2168-74.

12 Lafaurie $M$, Porcher $R$, Donay J-L, et al. Reduction of fluoroquinolone use is associated with a decrease in methicillin-resistant Staphylococcus aureus and fluoroquinolone-resistant Pseudomonas aeruginosa isolation rates: a 10 year study. J Antimicrob Chemother 2012;67:1010-15.

13 LeBlanc L, Pépin J, Toulouse K, et al. Fluoroquinolones and risk for methicillin-resistant Staphylococcus aureus, Canada. Emerg Infect Dis 2006:12:1398-405.

14 Aldeyab MA, Scott MG, Kearney MP, et al. Impact of an enhanced antibiotic stewardship on reducing methicillin-resistant Staphylococcus aureus in primary and secondary healthcare settings. Epidemiol Infect 2014;142:494-500.

15 Petrikkos G, Markogiannakis A, Papaparaskevas J, et al. Differences in the changes in resistance patterns to third- and fourth-generation cephalosporins and piperacillin/tazobactam among Klebsiella pneumoniae and Escherichia coli clinical isolates following a restriction policy in a Greek tertiary care hospita. Int J Antimicrob Agents 2007;29:34-8.

16 Aldeyab MA, Harbarth S, Vernaz N, et al. The impact of antibiotic use on the incidence and resistance pattern of extended-spectrum beta-lactamase-producing bacteria in primary and secondary healthcare settings. Br J Clin Pharmacol 2012;74:171-9.

17 Skrlin J, Bacic Vrca V, Marusic S, et al. Impact of ceftriaxone de-restriction on the occurrence of ESBL-positive bacterial strains and antibiotic consumption. J Chemother 2011;23:341-4.

18 Standiford HC, Chan S, Tripoli M, et al. Antimicrobial stewardship at a large tertiary care academic medical center: cost analysis before, during, and after a 7-year program. Infect Control Hosp Epidemiol 2012;33:338-45.

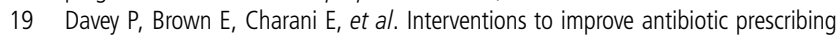
practices for hospital inpatients. Cochrane Database Syst Rev 2013;4:CD003543.

20 Robinson HL, Robinson PC, Whitby M. Poor Compliance with Community-Acquired Pneumonia Antibiotic Guidelines in a Large Australian Private Hospital Emergency 
Department. Microb Drug Resist Published Online First: 20 June 2014. doi:10.1089/ mdr.2014.0064

21 Kaur S, Rajagopalan S, Kaur N, et al. Drug utilization study in medical emergency unit of a tertiary care hospital in north India. Emerg Med Int 2014;2014:973578.

22 Pallin DJ, Camargo CA, Schuur JD. Skin infections and antibiotic stewardship: analysis of emergency department prescribing practices, 2007-2010. West J Emerg Med 2014;15:282-9.

23 Fagan $\mathrm{M}$, Lindbæk $\mathrm{M}$, Reiso $\mathrm{H}$, et al. A simple intervention to reduce inappropriate ciprofloxacin prescribing in the emergency department. Scand I Infect Dis 2014;46:481-5.

24 Schuetz P, Briel M, Christ-Crain M, et al. Procalcitonin to guide initiation and duration of antibiotic treatment in acute respiratory infections: an individual patient data meta-analysis. Clin Infect Dis 2012;55:651-62.

25 Schuetz P, Chiappa V, Briel M, et al. Procalcitonin algorithms for antibiotic therapy decisions: a systematic review of randomized controlled trials and recommendations for clinical algorithms. Arch Intern Med 2011;171:1322-31.

26 Schuetz P, Briel M, Mueller B. Clinical outcomes associated with procalcitonin algorithms to guide antibiotic therapy in respiratory tract infections. JAMA 2013:309:717-18.

27 Borde JP, Kaier K, Steib-Bauert M, et al. Feasibility and impact of an intensified antibiotic stewardship programme targeting cephalosporin and fluoroquinolone use in a tertiary care university medical center. BMC Infect Dis 2014;14:201.
28 Long $W$, Deng $X$, Zhang $Y$, et al. Procalcitonin guidance for reduction of antibiotic use in low-risk outpatients with community-acquired pneumonia. Respirology 2011;16:819-24.

29 Demonchy E, Dufour J-C, Gaudart J, et al. Impact of a computerized decision support system on compliance with guidelines on antibiotics prescribed for urinary tract infections in emergency departments: a multicentre prospective before-and-after controlled interventional study. J Antimicrob Chemother Published Online First: 4 June 2014. doi:10.1093/jac/dku191

30 Stein JC, Navab B, Frazee B, et al. A randomized trial of computer kiosk-expedited management of cystitis in the emergency department. Acad Emerg Med 2011;18:1053-9.

31 Ramsay C, Brown E, Hartman G, et al. Room for improvement: a systematic review of the quality of evaluations of interventions to improve hospital antibiotic prescribing. J Antimicrob Chemother 2003;52:764-71.

32 Wagner AK, Soumerai SB, Zhang F, et al. Segmented regression analysis of interrupted time series studies in medication use research. J Clin Pharm Ther 2002;27:299-309.

33 Ramsay CR, Matowe L, Grilli R, et al. Interrupted time series designs in health technology assessment: lessons from two systematic reviews of behavior change strategies. Int J Technol Assess Health Care 2003;19:613-23.

$34 \mathrm{http}: / /$ epoc.cochrane.org/sites/epoc.cochrane.org/files/uploads/21\%20Interrupted\% 20time\%20series\%20analyses $\% 202013 \% 2008 \% 2012$.pdf 